\title{
Mass corrections to the DGLAP equations
}

\author{
A. Jamali Hafshejani $\odot^{\dagger}$ and A. Mirjalili॰* \\ Physics Department, Yazd University, P.O. Box, 89195-741, Yazd, Iran
}

(Received 10 December 2020; accepted 16 February 2021; published 22 March 2021)

\begin{abstract}
We propose a mass-dependent momentum subtraction (MOM) scheme to renormalize UV divergence of unpolarized PDFs at one-loop order. This approach, which is based on a once subtracted dispersion relation, does not need a regulator. The overall counterterms are obtained from the imaginary part of the large transverse momentum region in loop integrals. The mass-dependent characteristic of the scheme yields to mass-dependent splitting functions for the DGLAP evolution equations. While the flavor number is fixed at any renormalization scale, the decoupling theorem is automatically imposed by the mass-dependent splitting functions. The required symmetries are also automatically respected by our prescription.
\end{abstract}

DOI: 10.1103/PhysRevD.103.054026

\section{INTRODUCTION}

The Wilson operator product expansion (OPE) $[1,2]$ provides a systematic approach to the factorization of quantum chromodynamics (QCD) [3,4], namely, the separation of contributions from long and short distances. The universal parton distribution functions (PDFs) are factorized from the hard partonic scattering. The latter can be calculated within perturbative QCD, but the PDFs have to be determined in a global analysis using experimental data $[5,6]$ and the Dokshitzer-Gribov-Lipatov-Altarelli-Parisi (DGLAP) equations [7-9] that provide the scale evolution of the PDFs.

In an alternative approach, the programs for the OPE can be performed in terms of matrix elements of gaugeinvariant nonlocal operators, giving an explicit definition of bare PDFs [10]. In this fashion, the bare PDFs are ultraviolet (UV) divergent in perturbation theory and require renormalization. The effective UV cutoff for the matrix elements is referred to as the factorization scale, separating the short distance from the PDFs. Consequently, the DGLAP equations appear as renormalization group equations (RGEs) for PDFs in which the kernels (AltarelliParisi splitting functions [9]) play the roles of anomalous dimensions of the PDFs. Following this approach, one comes up with different DGLAP equations in different renormalization schemes.

\footnotetext{
* Corresponding author. a.mirjalili@yazd.ac.ir

ahmad.jamalii86@gmail.com

Published by the American Physical Society under the terms of the Creative Commons Attribution 4.0 International license. Further distribution of this work must maintain attribution to the author(s) and the published article's title, journal citation, and DOI. Funded by SCOAP ${ }^{3}$.
}

Conventional minimal subtraction (MS) schemes, such as mass-independent types, could be appropriate choices if we were not to deal with heavy quarks. However, working within MS renormalization schemes, one encounters large logarithms of $m_{h} / \mu$ when $\mu \ll m_{h}$, where $m_{h}$ is a heavy quark mass and $\mu$ is the renormalization scale. To avoid this, one would follow an effective theory to decouple heavy quarks and absorb their mass effects in the renormalized coupling constant. An alternative approach is to take a composite scheme, using a different prescription to renormalize diagrams containing heavy quarks [proposed by Collins-Wilczek-Zee (CWZ) scheme [11] ]. However, requiring a definition of heavy quarks with respect to $\mu$ is inevitable. Unfortunately, heavy quark masses spread over a wide range of scales from a few to hundreds of GeVs. Consequently, we have to work with a series of subschemes, each of which considers a different number of heavy (decoupled) and light (active) quarks. Then, some definite thresholds should be considered to switch between the subschemes. In addition, matching conditions at the thresholds should be imposed. A number of such variable-flavor-number (VFN) schemes have been proposed for deep inelastic scattering (DIS) structure functions, including Aivazis-Collins-Olness-Tung (ACOT) [12,13], simplified ACOT (S-ACOT) [14,15], modified ACOT (ACOT- $\chi$ ) [16], Thorne-Roberts (TR) and its derivatives (TR') [17,18], and Fixed-Order-Next-toLeading-Log (FONLL) [19-21], etc. For a review on heavy quark mass effects in DIS and global analyses, see [22].

On the other hand, as discussed in [23], the critical issue of these schemes is that changes in the number of active quarks would lead to jumps in the splitting functions and the renormalized coupling constant. As a solution, a so-called "physical" factorization scheme is introduced in 
[23-25]. Providing mass-dependent splitting functions $[23,26]$, this scheme automatically results in a smooth behavior of PDFs, hard coefficient functions, and coupling constant. Although the idea of mass-dependent splitting functions had already been proposed in [27], the heavy quark part was included only at a large enough scale above the heavy quark mass. This approach gives irregularities at the heavy quark thresholds, and as shown in [28], the approach followed in [27] results in the same physical cross sections as those obtained by the ACOT scheme.

What we propose in this paper is a fixed-flavor-number, mass-dependent, momentum space subtraction (MOM) scheme based upon the analytic structure of the Feynman diagrams. Although mass-dependent MOM schemes decouple heavy quarks automatically and smoothly, they mostly suffer from complicated calculations and violation of symmetries of the theory in question. Therefore, defining a suitable mass-dependent MOM scheme requires careful considerations. Our scheme, on one hand, provides an automatically decoupling theorem, and on the other hand, it is lacking in possible disadvantages of a typical massdependent MOM scheme. All the required symmetries for PDFs, conservation of total momentum and flavor numbers, are automatically respected by our approach. Additionally, the possibility of extension to higher orders is supported by the provided computational simplifications. In our scheme, overall counterterms are extracted from off-shell Green functions of parton operators at large transverse momenta. This is carried out by a once subtracted dispersion relation (DR) due to the analytic properties of the Feynman diagrams.

The outcomes are mass-dependent splitting functions followed by mass corrections to the DGLAP equations. The MOM characteristic of our scheme implies that the decoupling theorem is imposed automatically and smoothly by mass correction terms in the splitting functions, while the flavor number is fixed. This scheme also yields the following computational simplifications: One only uses finite cut diagrams in a large transverse momenta region (full evaluation is not needed). Therefore, the requirement of any regulator vanishes.

This article is organized as follows: In Sec. II basic and required definitions of the unpolarized PDFs and the DGLAP equations are briefly reviewed. Then, we introduce our scheme in Sec. III. Some detailed calculations of renormalized Green functions are also given to clarify the prescription. In Sec. IV mass-dependent splitting functions are presented, and their features are discussed. The conclusion is given in Sec. V.

\section{DEFINITIONS}

We begin with a brief review on the theoretical framework of unpolarized PDFs and their renormalization. Bare PDFs can be defined as diagonal matrix elements of Fourier transformed of gauge-invariant bilocal light-ray operators [10]. For the quark type, one can write

$$
\begin{aligned}
O_{q_{i}}\left(k^{+}\right) \equiv & \int \frac{\mathrm{d} w^{-}}{4 \pi} \mathrm{e}^{-\mathrm{i} k^{+} w^{-}} \\
& \times: \bar{\psi}_{(0)}^{i}\left(n w^{-}\right) \gamma^{+} U_{\mathrm{F}}\left(u w^{-}, 0\right) \psi_{(0)}^{i}(0):,
\end{aligned}
$$

and for the gluon type, it is written as

$$
\begin{aligned}
O_{g}\left(k^{+}\right) \equiv & \int \frac{\mathrm{d} w^{-}}{-2 \pi k^{+}} \mathrm{e}^{-\mathrm{i} k^{+} w^{-}} \\
& \times: G_{(0)}^{+j}\left(n w^{-}\right) U_{\mathrm{A}}\left(u w^{-}, 0\right) G_{(0) j}^{+}(0):,
\end{aligned}
$$

where $\psi_{(0)}^{i}$ is the bare quark field of flavor $i$, and $G_{(0)}^{\mu \nu}$ is the bare field strength of the gluon. The sum over $j=1$ and 2 is understood in Eq. (2). The vector $u^{\mu} \equiv g_{-}^{\mu}$ points in the minus light-cone direction. We use the following definition for light-cone coordinates of a given vector $v$ :

$$
v^{ \pm} \equiv\left(v^{0} \pm v^{3}\right) / \sqrt{2} .
$$

The operator $U\left(u w^{-}, 0\right)$ is a Wilson line path ordered along $u[10]$ :

$U\left(u w^{-}, 0\right)=\mathcal{P} \exp \left[-\mathrm{i} g_{(0)} \int_{0}^{w^{-}} \mathrm{d} y^{-} A_{(0)}^{a,+}\left(u y^{-}\right) t_{a}\right]$,

where $A_{(0)}^{a, \mu}$ is the bare gauge field. Indices $F$ and $A$ in Eqs. (1) and (2) refer to "fundamental" and "adjoint" representations of SU(3) group generators $t_{a}$.

Consider a hadron $h$ moving in the $z$ direction with four momentum $P$ expressed by the $|P\rangle$ state. The probability of finding a parton of flavor $i$ with light-cone momentum fraction $\xi \equiv k^{+} / P^{+}$within the hadron $h$, as bare PDF, is given by the matrix element as follows:

$$
f_{(0) i / h}(\xi) \equiv\left\langle P\left|O_{i}\left(\xi P^{+}\right)\right| P\right\rangle_{\text {con }},
$$

where "con" implies that only connected diagrams should be taken into account.

The PDFs in Eq. (5) have an UV divergence that arises not only from the bare field operators but also the operator product in Eqs. (1) and (2). This requires us to apply a renormalization procedure using some renormalization factors and introducing a renormalization scale $\mu$ as

$$
f_{(\mathrm{R}) i / h}(\xi, \mu)=Z_{i j}(\xi, \mu) \otimes f_{(0) j / h}(\xi),
$$

where $\otimes$ is the conventional convolution in the $\xi$ parameter:

$$
g(\xi, \ldots) \otimes h(\xi, \ldots) \equiv \int_{\xi}^{1} \frac{\mathrm{d} x}{x} g(x, \ldots) h(\xi / x, \ldots) .
$$

At leading order accuracy, renormalization factors should be considered as

$$
Z_{i j}^{[0]}(\xi)=\delta_{i j} \delta(1-\xi)
$$

due to the fact that the renormalized and bare PDFs are the same at leading order. Notice the sum over $j$ in Eq. (6) runs over the gluon as well as all flavors of the quark and 
antiquark. Renormalization group equations for the renormalized PDFs imply the following DGLAP evolution equations,

$$
\mu^{2} \frac{\mathrm{d}}{\mathrm{d} \mu^{2}} f_{(\mathrm{R}) i / h}(\xi, \mu)=P_{i j}(\xi, \mu) \otimes f_{(\mathrm{R}) j / h}(\xi) .
$$

The kernels of the DGLAP equations are the anomalous dimension of renormalized PDFs and are referred to as splitting functions. They can be written in matrix form as

$$
P(\mu)=\mu^{2} \frac{\mathrm{d}}{\mathrm{d} \mu^{2}} \ln Z(\mu) .
$$

Therefore, splitting functions are actually scheme dependent.

Since the renormalization factors are independent of the hadron state, it would be convenient to replace the hadron state with a parton state, in the given definition of Eq. (5). This gives parton in parton distribution which can be carried out in the framework of perturbative QCD by means of the Lehmann-Symanzik-Zimmermann (LSZ) redundant formula [29]. Therefore, the bare unpolarized distribution of parton $i$ in quark $j$ reads

$$
f_{(0) i / j}(\xi)=\frac{1}{6} \lim _{p^{2} \rightarrow \bar{m}_{j}^{2}} \delta_{a b}\left(\not p+\bar{m}_{j}\right)_{\alpha \beta} \Gamma_{(0) i j}^{a b, \alpha \beta}\left(\xi p^{+}, p\right) .
$$

The factor $1 / 6$ is a consequence of the average over spins and colors of the target parton $j$. Here, $\Gamma_{(0) i j}$ is a bare amputated Green function of the parton operator $O_{i}$, defined in Eq. (1) or (2), accompanied by two field operators $\psi_{(0)}^{j}$ and $\bar{\psi}_{(0)}^{j}$ with off-shell external momenta $p$ and $-p$ :

$\Gamma_{(0) i j}^{a b, \alpha \beta}\left(k^{+}, p\right) \equiv\left\langle 0\left|\mathcal{T} O_{i}\left(k^{+}\right) \psi_{(0) j}^{a, \alpha}(p) \bar{\psi}_{(0) j}^{b, \beta}(-p)\right| 0\right\rangle_{\mathrm{amp}}$,

where "amp' refers to the "amputated" Green function and $\bar{m}$ is the physical mass. Note that Fourier transformations are defined outside the time order operator. We work with bare fields (instead of physical ones) for which loops on external lines should be taken into account. Note that the Green function in Eq. (12) is time ordered, which gives the same result as the fixed ordered one as long as we work in a covariant gauge [30]. A coordinate system is chosen such that the external momentum has no transverse component,

$$
p^{\mu}=g_{+}^{\mu} p^{+}+g_{-}^{\mu} p^{2} / 2 p^{+},
$$

where $g_{\mu \nu}$ is the metric tensor.

In the same manner, the bare unpolarized distribution of a parton $i$ in a gluon can be written in terms of an amputated Green function as

$$
f_{(0) i / g}(\xi)=\frac{1}{16} \lim _{p^{2} \rightarrow \bar{m}_{j}^{2}} \delta_{a b} d_{\mu \nu}(p) \Gamma_{(0) g j}^{a b, \mu \nu}\left(\xi p^{+}, p\right),
$$

where the amputated Green function reads

$$
\Gamma_{(0) i g}^{a b, \mu \nu}\left(k^{+}, p\right) \equiv\left\langle 0\left|\mathcal{T} O_{i}\left(k^{+}\right) A_{(0)}^{a, \mu}(p) A_{(0)}^{b, \nu}(-p)\right| 0\right\rangle_{\mathrm{amp}} .
$$

The sum over physical polarizations in Eq. (14) is done by following tensor:

$$
d_{\mu \nu}(p) \equiv-g_{\mu \nu}+\frac{p_{\mu} g_{\nu}^{+}+p_{\nu} g_{\mu}^{+}}{p^{+}} .
$$

The factor 1/16 in Eq. (14) results from the average over physical polarizations and colors of the initial gluon.

From Eqs. (12) and (14), a renormalized parton in the parton distributions can be defined by replacing the bare amputated Green functions with their renormalized version. On the other hand, regarding the standard definition given by Eq. (6), one can consider the same renormalization relation for the Green functions as

$\Gamma_{(\mathrm{R}) i j}\left(\xi p^{+}, p ; \mu\right)=Z_{i k}(\xi, \mu) \otimes \Gamma_{(0) k j}\left(\xi p^{+}, p\right)+\ldots$,

where the dots indicate contributions of the Green functions of possible non-gauge-invariant [but Becchi-Rouet-StoraTyutin (BRST) invariant] operators mixing with the parton operators in the renormalization process [31]. These unphysical contributions, however, are not our concern as long as they are eliminated by the equations of motion imposed by the Lehmann-Symanzik-Zimmermann (LSZ) redundant formula. Therefore, these unphysical Green functions do not contribute to the PDF renormalization. In general, renormalization factors may carry tensor and spinor indices contracting with Green functions, but we assume that, in an appropriate scheme, they appear to be scalar coefficients. This is justified in our scheme in Sec. III for the cases in which the target is a quark. According to this assumption, renormalization factors introduced in Eqs. (6) and (17) turn out to be equal.

\section{OUR PROPOSED RENORMALIZATION SCHEME}

Since the PDFs are supposed to contain information about the long-distance properties of hadrons, a renormalization program is needed to remove the short-distance behavior of the bare PDFs. Therefore, we introduce the following renormalization condition on a given diagram, $\Gamma^{(\gamma)}$ :

$$
\Gamma_{(\mathrm{R})}^{(\gamma)}\left(p^{2} ; \mu\right)=\Gamma^{(\gamma)}\left(p^{2}\right)-\Gamma_{\mathrm{UV}}^{(\gamma)}\left(p^{2}=-\mu^{2}\right),
$$

from which only the UV-divergent part is subtracted.

To clarify our formalism, let us first consider a dispersion relation (DR). Given the imaginary part of an analytic function $f(x)$, one can write a DR as 


$$
f(x)=\int_{x_{\min }}^{\infty} \frac{\mathrm{d} s}{s-x-\mathrm{i} \epsilon} \frac{\operatorname{Im} f(s)}{\pi} .
$$

If the integral is logarithmically divergent, a once subtracted DR can be performed to obtain a finite result

$f(x)-f(c)=\int_{x_{\min }}^{\infty} \mathrm{d} s \frac{x-c}{(s-x-\mathrm{i} \epsilon)(s-c)} \frac{\operatorname{Im} f(s)}{\pi}$,

where $c<x_{\min }$ to avoid singularity.

Therefore, the condition introduced in Eq. (18) can be carried out by using a once subtracted DR, Eq. (20), in which the total imaginary part is replaced by a particular piece of the imaginary part generating just the UV divergence of a given diagram:

$$
\begin{aligned}
& \Gamma_{(\mathrm{R})}^{(\gamma)}\left(p^{2} ; \mu\right) \\
& =\int_{p_{\min }^{2}}^{\infty} \mathrm{d} s \frac{s+\mu^{2}}{\left(s-p^{2}-\mathrm{i} \epsilon\right)\left(s+\mu^{2}\right)} \frac{\mathrm{UIm}^{(\gamma)}(s)}{\pi}+\Gamma_{\mathrm{IR}}^{(\gamma)}\left(p^{2}\right),
\end{aligned}
$$

where "UIm" is defined as the "particular term of the imaginary part that generates the UV divergence." In Eq. (21), just the UV divergence is subtracted, and the UV-finite part, indicated by $\Gamma_{\mathrm{IR}}^{(\gamma)}$, is left as is. In our proposed renormalization scheme, therefore, one is supposed to find just the UIm of a diagram instead of its total imaginary part. This can simply be done by using cut diagrams in the UV region of the loop integral, i.e., the limit of infinite transverse momenta. Note that a sum over all possible legitimate cut versions of a given diagram amounts to its total imaginary part [32],

$$
\operatorname{Im} \Gamma^{(\gamma)}=\frac{1}{2} \sum_{\text {cut }} \Gamma_{\text {cut }}^{(\gamma)}
$$

Since, in general, UV-finite terms in counterterms do not automatically satisfy the required symmetries, over-subtraction may be needed to impose required constraints on the counterterms. However, as long as our prescription avoids these UV-finite terms, the renormalized PDFs automatically satisfy the required constraints. This is shown by explicit calculations in the following, and the resulting splitting functions respect all the required symmetries.

It should be noted that not only is calculating the total imaginary part of a diagram more complicated in comparison to the UIm, but the total imaginary part may also be singular in general. Requiring just the UIm of diagrams, our prescription, therefore, is followed by the following two simplifications:

1. The UV limit of a cut diagram should be calculated instead of its total value.

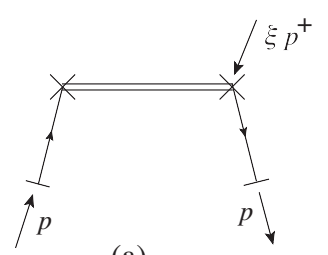

(a)

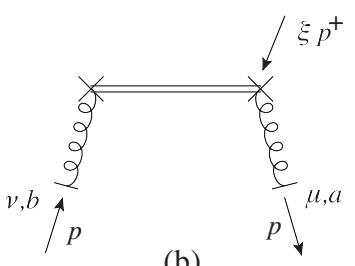

(b)
FIG. 1. Cut diagrams of (a) quark in quark and (b) gluon in gluon amputated Green functions at leading order.

2. There is no need for a regulator since the UIm is finite.

In the rest of this section, we calculate the UIm of the amputated Green functions to renormalize them by using Eq. (21). It is worth mentioning that all the cut diagrams presented here would vanish if the external momenta were on shell.

At leading order, we have two nonvanishing Green functions: quark in quark type, Fig. 1(a), and gluon in gluon type, Fig. 1(b). Thus, at leading order, we have

$$
\Gamma_{i j, a b}^{[0] \alpha \beta}\left(\xi k^{+}, p\right)=\frac{\gamma_{\alpha \beta}^{+}}{2 p^{+}} \sigma\left(p^{+}\right) \delta_{a b} \delta_{i j} \delta(1-\xi),
$$

and

$\Gamma_{g g a b}^{[0] \mu \nu}\left(\xi k^{+}, p\right)=g^{\mu i} g_{i}^{\nu} \delta_{a b} \sigma\left(p^{+}\right)[\delta(1-\xi)-\delta(1+\xi)]$,

where $\sigma$ indicates the sign function. From now on, we consider a particle in particle distributions, i.e., for which $p^{+}$and $k^{+}>0$, and suppress flavor and color indices (as long as they appear only in Kronecker delta indices). Spinor indices, which are explicit from $\gamma$-matrices, are also suppressed. It is convenient to indicate the dependence of $k^{+}$through the $\xi=k^{+} / p^{+}$ratio.

Substituting the leading-order Green functions and renormalization factors-Eqs. (23), (24), and (8)-in Eq. (17), one gets the following expressions at one-loop order:

$$
\begin{aligned}
& \Gamma_{(\mathrm{R}) q q}^{[1]}(\xi, p ; \mu)=\Gamma_{(0) q q}^{[1]}(\xi, p)+\frac{\gamma^{+}}{2 p^{+}} Z_{q q}^{[1]}(\xi, \mu), \\
& \Gamma_{(\mathrm{R}) g q}^{[1]}(\xi, p ; \mu)=\Gamma_{(0) g q}^{[1]}(\xi, p)+\frac{\gamma^{+}}{2 p^{+}} Z_{g q}^{[1]}(\xi, \mu), \\
& \Gamma_{(\mathrm{R}) q g}^{[1] \mu \nu}(\xi, p ; \mu)=\Gamma_{(0) q g}^{[1] \mu \nu}(\xi, p)+g^{\mu i} g_{i}^{\nu} Z_{q g}^{[1]}(\xi, \mu) .
\end{aligned}
$$

We start with the most important case of quark in quark. Detailed calculations are represented in order to clarify our prescription. Consider cut diagrams of quark in quark PDF as in Fig. 2. The total value of diagrams (a) and its Hermitian conjugate $\left(\mathrm{a}^{\dagger}\right)$, indicated by "h.c." in Fig. 2(a), are exactly the UIm, which gives 

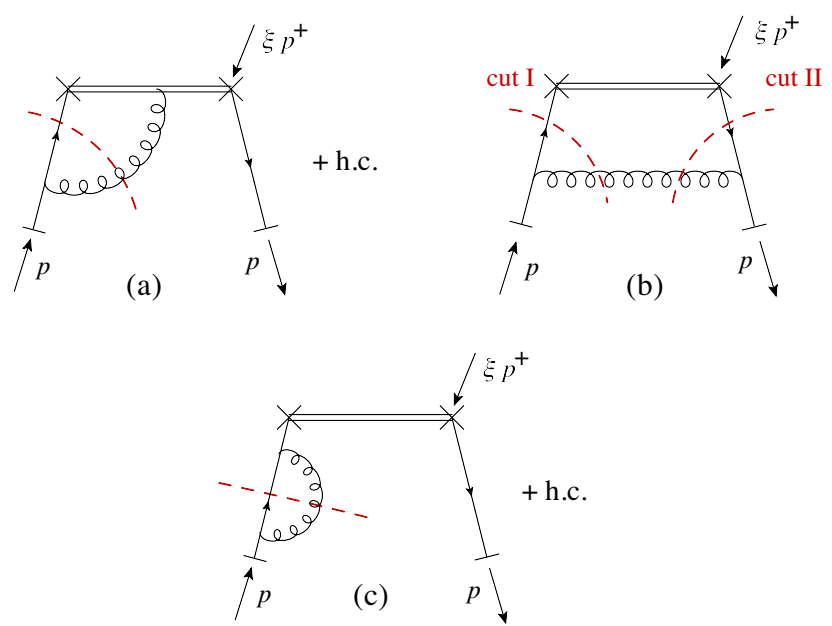

FIG. 2. Cut diagrams of a quark in quark amputated Green function at one-loop order.

$\frac{1}{2} \Gamma_{\text {cut }}^{\left(\mathrm{a}+\mathrm{a}^{\dagger}\right)}(\xi, p)=\frac{g^{2}}{16 \pi} C_{\mathrm{F}} \frac{\gamma^{+}}{p^{+}}\left[\frac{2 \xi}{1-\xi} \theta\left(p^{2}-m^{2} / \xi\right)\right]_{+}$,

where a plus distribution is defined as

$$
[f(\xi)]_{+} \equiv f(\xi)-\theta(0<\xi<1) \delta(1-\xi) \int_{0}^{1} \mathrm{~d} \alpha f(\alpha),
$$

and $C_{\mathrm{F}}$ is the value of the quadratic Casimir operator of the $\mathrm{SU}(3)$ group in the fundamental representation. On the other hand, for the case of the cut diagram in Fig. 2(b), we need to extract the UIm from the total imaginary part which is given by

$$
\begin{aligned}
& \frac{1}{2}\left(\Gamma_{\text {cut I }}^{(\mathrm{b})}+\Gamma_{\text {cut II }}^{(\mathrm{b})}\right)(\xi, p) \\
& =\frac{-g^{2}}{16 \pi} C_{\mathrm{F}}(1-\xi) \theta(0<\xi<1) \\
& \quad \times \int_{0}^{\infty} \mathrm{d} \mathbf{q}_{\mathbf{T}}^{2} \frac{\mathbf{q}_{\mathbf{T}}^{2} \frac{\gamma^{+}}{p^{+}}+2 \xi^{2} p^{+} \gamma^{-}-4 m \xi}{\mathbf{q}_{\mathbf{T}}^{2}+M\left(\xi, p^{2}\right)} \delta\left(\mathbf{q}_{\mathbf{T}}^{2}+M\left(\xi, p^{2}\right)\right),
\end{aligned}
$$

where $M\left(\xi, p^{2}\right) \equiv(1-\xi)\left(m^{2}-\xi p^{2}\right)$. As mentioned above, the UIm can be derived from the UV region of the integral, i.e., $\mathbf{q}_{\mathbf{T}}^{\mathbf{2}} \rightarrow \infty$. Therefore, we get

$$
\begin{aligned}
\operatorname{UIm} \Gamma^{(\mathrm{b})}(\xi, p)= & -\frac{g^{2}}{16 \pi} C_{\mathrm{F}} \frac{\gamma^{+}}{p^{+}}(1-\xi) \\
& \times \theta(0<\xi<1) \theta\left(p^{2}-m^{2} / \xi\right) .
\end{aligned}
$$

The contribution of diagrams (c) and $\left(\mathrm{c}^{\dagger}\right)$ in Fig. 2 amounts to

$$
\begin{aligned}
\frac{1}{2} \Gamma_{\text {cut }}^{\left(\mathrm{c}+\mathrm{c}^{\dagger}\right)}(\xi, p)= & \frac{g^{2}}{16 \pi} \int_{0}^{1} \mathrm{~d} \alpha(1-\alpha) \int_{0}^{+\infty} \mathrm{d} \mathbf{q}_{\mathbf{T}}^{2} \delta\left(\mathbf{q}_{\mathbf{T}}^{\mathbf{2}}+M\left(\alpha, p^{2}\right)\right) \\
& \times \frac{\left(\mathbf{q}_{\mathbf{T}}^{2}-m^{2}\right) \frac{\gamma^{+}}{p^{+}}+\alpha(\alpha-2) m^{2}}{\mathbf{q}_{\mathbf{T}}^{2}-(1-\alpha)^{2} m^{2}}
\end{aligned}
$$

where the UV limit of the integral gives the associated UIm as

$\mathrm{UIm} \Gamma^{\left(\mathrm{c}+\mathrm{c}^{\dagger}\right)}(\xi, p)=\frac{g^{2}}{16 \pi} C_{\mathrm{F}} \frac{\gamma^{+}}{p^{+}} \int_{0}^{1} \mathrm{~d} \alpha(1-\alpha) \theta\left(p^{2}-m^{2} / \xi\right)$.

Therefore, by substituting the total UIm in Eq. (21), the renormalized Green function of the quark in quark amounts to

$$
\begin{aligned}
& \Gamma_{(\mathrm{R}) q q}^{[1]}(\xi, p ; \mu) \\
& =-\frac{g^{2}}{16 \pi^{2}} C_{\mathrm{F}} \frac{\gamma^{+}}{p^{+}}\left[\frac{1+\xi^{2}}{1-\xi} \int_{\frac{m^{2}}{\xi}}^{\infty} \frac{\mathrm{d} s}{s-p^{2}} \frac{\mu^{2}+p^{2}}{\mu^{2}+s}\right]_{+} \\
& \quad+\Gamma_{\mathrm{IR}}(\xi, p) .
\end{aligned}
$$

Notice the pure plus distribution form of the counterterm, resulting in conservation of each flavor number. The renormalized Green function, Eq. (34), is identical to subtracting the logarithm part of the bare one at the renormalization point $-\mu^{2}$ as well as the pole part of a regulator. Therefore, as opposed to a MS scheme, we do not have such a large logarithm of $m / \mu$ here. To avoid these large logarithms in a MS scheme, either one should get heavy quarks decoupled at low renormalization scales, $\mu \ll m$, or use a mass-dependent scheme for diagrams involving heavy quarks (like the CWZ scheme [11]).

The same approach is applied to the other cases. There is one diagram with two cuts for the Green function of the gluon in quark, depicted in Fig. 3. Evaluated at large transverse momenta, the cut diagrams give the UIm as

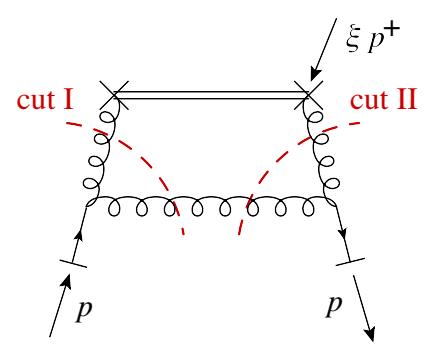

FIG. 3. Cut diagrams of the gluon in quark amputated Green function at one-loop order. 


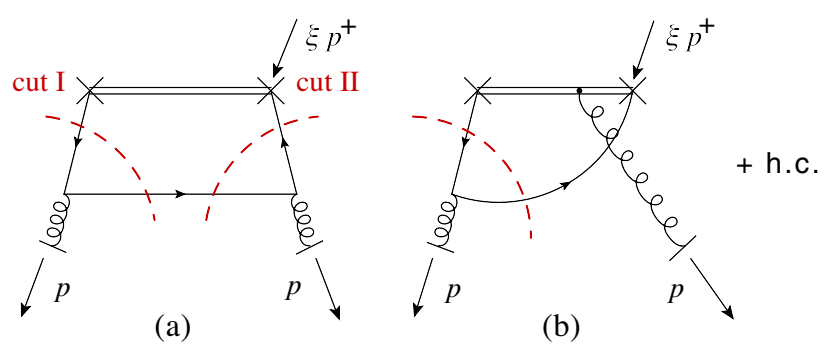

FIG. 4. Cut diagrams of the quark in gluon amputated Green function at one-loop order.

$$
\begin{aligned}
& \operatorname{UIm} \Gamma_{(0) g q}^{[1]}(\xi, p ; \mu) \\
& \quad=-\frac{g^{2}}{8 \pi} C_{\mathrm{F}} \frac{\gamma^{+}}{p^{+}} \frac{2-2 \xi+\xi^{2}}{\xi} \theta\left(p^{2}-\frac{m^{2}}{\xi(1-\xi)}\right) .
\end{aligned}
$$

Notice the transformation $\xi \rightarrow 1-\xi$ under which the counterterm of the gluon in quark Green function transforms to the counterterm of the quark in quark Green function. This manifests the conservation of total momentum.

In general, we have three cut diagrams associated with the case of the quark in gluon, illustrated in Fig. 4. To get rid of diagram (b), we can project the Green function by $d_{\mu \nu}(p)$, defined in Eq. (16). By so doing, we actually sum over physical polarizations, which results in extracting renormalization factors for an unpolarized target gluon:

$Z_{q g}^{[1]}(\xi, \mu)=\frac{1}{2}\left[d_{\mu \nu}(p) \Gamma_{q g}^{(a) \mu \nu}(\xi, p)-d_{\mu \nu}(p) \Gamma_{(\mathrm{R}) q g}^{(a) \mu \nu}(\xi, p ; \mu)\right]$

Therefore, to find the renormalization factor, we just need to obtain the UIm of the projected diagram (a), which would be

$$
\begin{aligned}
& \mathrm{UIm}\left[d_{\mu \nu}(p) \Gamma_{q g}^{(a) \mu \nu}(\xi, p)\right] \\
& \quad=-\frac{g^{2}}{8 \pi} T_{\mathrm{R}}\left(2 \xi^{2}-2 \xi+1\right) \theta\left(p^{2}-\frac{m^{2}}{\xi(1-\xi)}\right),
\end{aligned}
$$

where $T_{\mathrm{R}}$ is conventional notation for the normalization of the $\mathrm{SU}(3)$ group generators.

Note that, in our scheme, the renormalization factors of the antiquark have the following symmetries:

$$
\begin{aligned}
& Z_{q q}(\xi, \mu)=Z_{\bar{q} \bar{q}}(\xi, \mu), \\
& Z_{\bar{q} q}(\xi, \mu)=Z_{q \bar{q}}(\xi, \mu),
\end{aligned}
$$

and

$$
Z_{q g}(\xi, \mu)=Z_{\bar{q} g}(\xi, \mu)=Z_{q g}(1-\xi, \mu)
$$

The latter symmetry guarantees that the renormalized nonsinglet distribution,

$$
f_{(\mathrm{R}) q / h}^{\mathrm{NS}}(\xi, \mu) \equiv f_{(\mathrm{R}) q / h}(\xi, \mu)-f_{(\mathrm{R}) \bar{q} / h}(\xi, \mu),
$$

does not mix with the gluon distribution. Moreover, the combination of all the symmetries given by Eqs. (38)-(40) gives an explicit definition for the renormalization factor of a nonsinglet distribution as

$$
f_{(\mathrm{R}) q / h}^{\mathrm{NS}}(\xi, \mu)=\left[Z_{q q}(\xi, \mu)-Z_{q \bar{q}}(\xi, \mu)\right] \otimes f_{(0) q / h}^{\mathrm{NS}}(\xi) .
$$

\section{MASS-DEPENDENT SPLITTING FUNCTIONS}

In this section, we represent the mass-dependent splitting functions obtained by the scheme introduced in this paper. In general, the resulting splitting functions are proportional to the conventional ones multiplied by a mass-dependent coefficient. We show that these coefficients automatically impose a smooth decoupling on the DGLAP equations.

As mentioned, splitting functions are the anomalous dimension of the parton in parton PDFs, Eq. (10). Thus, at one-loop order, they can be derived by

$$
\begin{aligned}
P_{i j}^{[1]}(\xi, \mu) & =\mu^{2} \frac{\mathrm{d}}{\mathrm{d} \mu^{2}} Z_{(\mathrm{R}) i j}^{[1]}(\xi, p ; \mu) \\
& =-\mu^{2} \frac{\mathrm{d}}{\mathrm{d} \mu^{2}} \Gamma_{(\mathrm{R}) i j}^{[1]}(\xi, p ; \mu),
\end{aligned}
$$

in our approach. Substituting the renormalized Green functions, e.g., Eq. (34), in Eq. (43), we obtain the mass-dependent splitting functions as follows:

$$
\begin{gathered}
P_{q q}^{[1]}(\xi, r)=\frac{g^{2}}{8 \pi^{2}} C_{\mathrm{F}}\left[\frac{1+\xi^{2}}{1-\xi} \frac{\xi}{r+\xi}\right]_{+}, \\
P_{g q}^{[1]}(\xi, r)=\frac{g^{2}}{8 \pi^{2}} C_{\mathrm{F}}\left[\frac{1+(1-\xi)^{2}}{\xi} \frac{1-\xi}{r+1-\xi}\right],
\end{gathered}
$$

and

$P_{q g}^{[1]}(\xi, r)=\frac{g^{2}}{8 \pi^{2}} T_{\mathrm{R}}\left[1-\left(\frac{r}{r+\xi(1-\xi)}\right)^{2}\right]\left[\xi^{2}+(1-\xi)^{2}\right]$,

where $r \equiv m^{2} / \mu^{2}$. The conservation of quark number for each flavor implies that $P_{q q}$ should be in the form of a plus distribution, which is automatically satisfied in our scheme. In addition, the conservation of the total momentum results in the symmetry

$$
P_{g q}(1-\xi, r)=P_{q q}(\xi, r)
$$


which is also automatically respected in our scheme and in the "physical" scheme in [26]. It is worth mentioning that the conventional symmetry

$$
P_{q g}(1-\xi, r)=P_{q g}(\xi, r)
$$

also shows up in our mass-dependent splitting function. This symmetry actually originates from the symmetry given by Eq. (40). By violating this symmetry, the nonsinglet distribution, defined by Eq. (41), mixes with the gluon distribution in the DGLAP equation. This symmetry, however, is not respected in the mass-dependent splitting function $P_{q g}$ represented in [23].

Mass correction to the conventional $P_{g g}$ splitting function, at one-loop order, should be performed on the coefficient of the $T_{R}$ in the delta function coefficient. This term is responsible for fermionic loops in the external gluon propagator. The mass correction can be determined using the sum rule

$$
\int_{0}^{1} \mathrm{~d} \xi \xi P_{g g}(\xi)+\sum_{i}^{6} \int_{0}^{1} \mathrm{~d} \xi \xi\left[P_{q_{i} g}\left(\xi, r_{i}\right)+P_{\bar{q}_{i} g}\left(\xi, r_{i}\right)\right]=0,
$$

which results from the conservation of total momentum. Substituting the mass-dependent splitting function $P_{q g}$, Eq. (46), in the sum-rule given by Eq. (49), we obtain the mass correction to the conventional splitting function $P_{g g}$, which is a replacement of the flavor number $n_{f}$ in the conventional $P_{g g}$ with the summation

$$
\sum_{i=1}^{6} \pi\left(r_{i}\right)
$$

where

$\pi(r) \equiv \frac{1}{1+4 r}\left[1+r-6 r^{2}+\frac{12 r^{3}}{\sqrt{1+4 r}} \ln \frac{\sqrt{1+4 r}+1}{\sqrt{1+4 r}-1}\right]$.

Note that the flavor number is fixed at 6 in Eq. (50).

Having a smooth behavior across the heavy-quark thresholds, the function Eq. (51) is analogous to the step function $\theta\left(\mu-\mu_{\text {threshold }}\right)$ in VFN schemes. The plot in Fig. 8 shows that $\pi(r)$ behaves like a very smooth step function, and it smoothly adds the contribution of each heavy-quark loop in gluon self-energy from low to high scales. The contribution for each heavy-quark flavor is completely decoupled at renormalization scales much lower than the quark mass. For renormalization scales much higher than the quark mass, the function $\pi(r)$ acts as if the quark were massless, as evidenced in Fig. 8.
In addition to the function $\pi(r)$, decoupling heavy quarks in the DGLAP equations is also controlled by the mass-dependent coefficients of the splitting functions in Eq. (44)-(46) automatically. Plots depicted in Figs. 5-7 obviously reveal the fact that the mass-dependent splitting functions are strongly suppressed by the large values $r \gg 1$ and approach to the conventional ones at small values $r \ll 1$. This justifies the fact that, at any renormalization scale, one should sum over all six quark and antiquark flavors in the DGLAP equations when using these massdependent splitting functions. They automatically control the effects of heavy-quark masses in the DGLAP equations

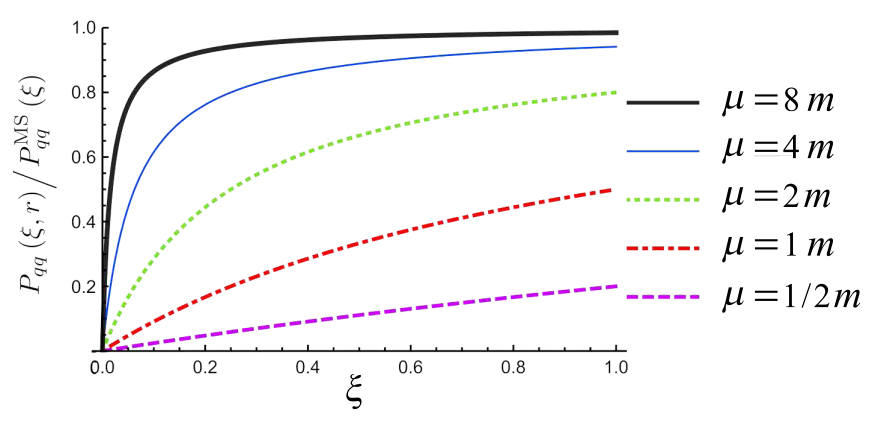

FIG. 5. Effect of the mass-dependent coefficient on the splitting function $P_{q q}$.

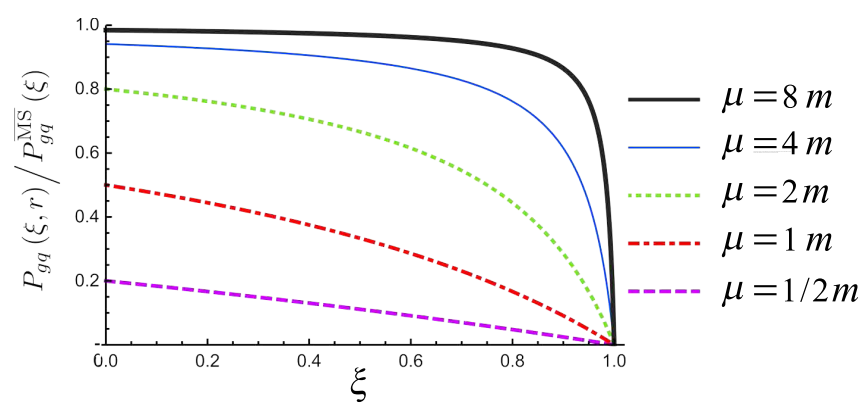

FIG. 6. Effect of the mass-dependent coefficient on the splitting function $P_{g q}$.

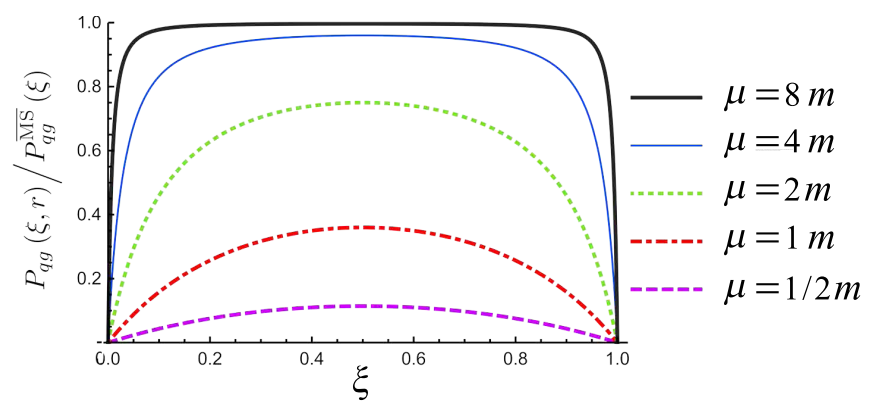

FIG. 7. Effect of the mass-dependent coefficient on the splitting function $P_{q g}$. 


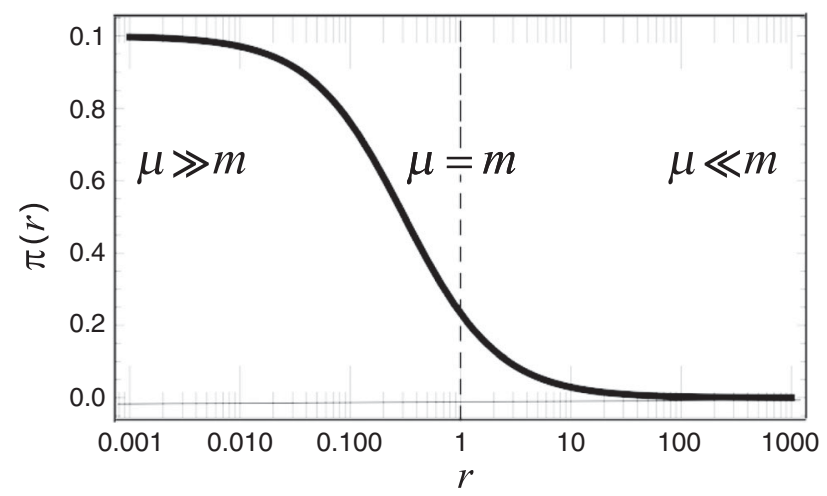

FIG. 8. Function $\pi(r)$ which gives the contribution of each heavy-quark loop to $P_{g g}$.

with respect to the renormalization scale, starting from an input scale $Q_{0}$.

The difference between the splitting functions introduced in this work and in $[23,26]$ emphasizes the fact that splitting functions are scheme dependent. The output is such that our splitting functions do not contain an additive term, in contrast to $[23,26]$. Our approach automatically respects symmetries since we define counterterms as lacking in the UV-finite part. However, the splitting functions obtained by use of a so-called physical scheme in [23] violate symmetries given by Eqs. (47) and (48), but the former symmetry is recovered in [26]. This violation shows that the counterterms determined by the physical scheme contain symmetry-breaking terms.

In addition to the scale region at which the mass correction has an influential contribution, the dominant region of momentum fraction $\xi$ at which the mass correction plays a substantial role can obviously be identified from plots of the mass-dependent coefficients. Plots of Fig. 5 show that the mass correction suppresses the possibility that a heavy quark, with a small momentum fraction, splits from a heavy parent quark. In other words, since we have the symmetry given in Eq. (47), the emission of a soft gluon from a heavy quark is still possible at relatively low scales, while the possibility of gluon emission with a large momentum fraction from a heavy quark is suppressed at even scales $\mu \simeq m$ (which can also be realized from Fig. 6).
On the other hand, from the mass-dependent coefficient of $P_{g q}$ illustrated in Fig. 7, we can infer that the mass correction to $P_{g q}$ is mostly suppressed when the magnitude of $\xi(1-\xi)$ is small. This can be interpreted as follows: A gluon is more likely to split into a quark and antiquark pair with almost the same momentum fractions, $\xi \simeq 1 / 2$, in the case of heavy quarks.

\section{CONCLUSION}

In this work, we use a mass-dependent MOM scheme to renormalize unpolarized PDFs. Using this unconventional approach, we subtract large transverse momenta behavior (as well as the UV pole part) of bare PDFs, which actually belong to the hard region. A once subtracted dispersion relation is applied to perform the subtraction using the imaginary part of the Feynman diagrams. We define the specific part of the total imaginary part corresponding to only the UV divergence in the real part. This approach is followed by computational simplifications and automatically respecting symmetries. The resulting splitting functions differ from the conventional ones by a mass-dependent coefficient. We show that these coefficients automatically decouple heavy quarks in the DGLAP equations. Therefore, the flavor number is fixed at 6 at any renormalization scale, and decoupling is automatically imposed, very smoothly, by the mass-dependent splitting functions.

Some phenomenological work is needed to test the prescription. The PDF of the gluon and light quarks at a low initial scale would be taken as the input for the DGLAP equations. The input heavy-quark PDFs would be set to zero or treated as "intrinsic" PDFs as introduced in [33], and the summation in the DGLAP equations should run over all 6 quark and antiquark flavors. The results would specifically show a nonzero contribution for the evolution of heavy-quark PDFs at renormalization scales below their masses. This contribution, however, would not be of large magnitude since not only are the mass-dependent coefficients of splitting functions highly suppressed at renormalization scales $\mu \ll m$, but they also remain small at even scales $\mu \simeq m$, as can be seen in Figs. 5-7. Therefore, we would have a very smooth evolution of each heavy-quark PDF starting somewhere in the region $\mu \simeq m$.
[1] K. G. Wilson, Non-Lagrangian models of current algebra, Phys. Rev. 179, 1499 (1969).

[2] R. A. Brandt and G. Preparata, Operator product expansions near the light cone, Nucl. Phys. B27, 541 (1971).
[3] J. C. Collins, D. E. Soper, and G. F. Sterman, Factorization of hard processes in QCD, Adv. Ser. Dir. High Energy Phys. 5, 1 (1989).

[4] J. C. Collins, Hard scattering factorization with heavy quarks: A general treatment, Phys. Rev. D 58, 094002 (1998). 
[5] A. Accardi et al., A critical appraisal and evaluation of modern PDFs, Eur. Phys. J. C 76, 471 (2016).

[6] D. Clark et al. (nCTEQ Collaboration), nCTEQ PDFs at the LHC: Vector boson production in heavy ion collisions, Proc. Sci., DIS2019 (2019) 024 [arXiv:1909.00452].

[7] Y. L. Dokshitzer, Calculation of the structure functions for deep inelastic scattering and $e^{+} e^{-}$annihilation by perturbation theory in quantum chromodynamics, Sov. Phys. JETP 46, 641 (1977).

[8] V. Gribov and L. Lipatov, Deep inelastic e p scattering in perturbation theory, Sov. J. Nucl. Phys. 15, 438 (1972).

[9] G. Altarelli and G. Parisi, Asymptotic freedom in parton language, Nucl. Phys. B126, 298 (1977).

[10] J. C. Collins and D. E. Soper, Parton distribution and decay functions, Nucl. Phys. B194, 445 (1982).

[11] J. C. Collins, F. Wilczek, and A. Zee, Low-energy manifestations of heavy particles: Application to the neutral current, Phys. Rev. D 18, 242 (1978).

[12] M. Aivazis, F. I. Olness, and W.-K. Tung, Leptoproduction of heavy quarks. 1. General formalism and kinematics of charged current and neutral current production processes, Phys. Rev. D 50, 3085 (1994).

[13] M. Aivazis, J. C. Collins, F. I. Olness, and W.-K. Tung, Leptoproduction of heavy quarks. 2. A unified QCD formulation of charged and neutral current processes from fixed target to collider energies, Phys. Rev. D 50, 3102 (1994).

[14] J. C. Collins, Proof of factorization for diffractive hard scattering, Phys. Rev. D 57, 3051 (1998); Erratum, Phys. Rev. D 61, 019902 (1999).

[15] M. Krämer, F. I. Olness, and D. E. Soper, Treatment of heavy quarks in deeply inelastic scattering, Phys. Rev. D 62 , 096007 (2000).

[16] W.-K. Tung, S. Kretzer, and C. Schmidt, Open heavy flavor production in QCD: Conceptual framework and implementation issues, J. Phys. G 28, 983 (2002).

[17] R. Thorne and R. Roberts, An ordered analysis of heavy flavor production in deep inelastic scattering, Phys. Rev. D 57, 6871 (1998).

[18] R. Thorne, A variable-flavor number scheme for NNLO, Phys. Rev. D 73, 054019 (2006).

[19] M. Buza, Y. Matiounine, J. Smith, and W. van Neerven, Charm electroproduction viewed in the variable flavor number scheme versus fixed order perturbation theory, Eur. Phys. J. C 1, 301 (1998).
[20] M. Cacciari, M. Greco, and P. Nason, The P(T) spectrum in heavy flavor hadroproduction, J. High Energy Phys. 05 (1998) 007.

[21] S. Forte, E. Laenen, P. Nason, and J. Rojo, Heavy quarks in deep-inelastic scattering, Nucl. Phys. B834, 116 (2010).

[22] R. Thorne and W. Tung, PQCD formulations with heavy quark masses and global analysis, in HERA and the LHC, Proceedings of the Workshop HERA and the LHC, (Hamburg Dt. Elektronen-Synchrotron, 2009), pp. 332-351 [arXiv:0809.0714].

[23] E. de Oliveira, A. Martin, M. Ryskin, and A. Shuvaev, Treatment of heavy quarks in QCD, Eur. Phys. J. C 73, 2616 (2013).

[24] E. Oliveira, A. Martin, and M. Ryskin, Physical factorisation scheme for PDFs for non-inclusive applications, J. High Energy Phys. 11 (2013) 156.

[25] E. Oliveira, A. Martin, and M. Ryskin, Treatment of the infrared contribution: NLO QED evolution as a pedagogic example, Eur. Phys. J. C 73, 2534 (2013); , Erratum, Eur. Phys. J. C 73, 2641 (2013).

[26] A. D. Martin and M. G. Ryskin, Comments on global parton analyses, Acta Phys. Pol. B 51, 1079 (2020).

[27] A. D. Martin, R. Roberts, M. Ryskin, and W. Stirling, Consistent treatment of charm evolution in deep inelastic scattering, Eur. Phys. J. C 2, 287 (1998).

[28] F. I. Olness and R. J. Scalise, Heavy quark parton distributions: Mass dependent or mass independent evolution?, AIP Conf. Proc. 407, 320 (1997).

[29] H. Lehmann, K. Symanzik, and W. Zimmermann, On the formulation of quantized field theories, Nuovo Cimento 1, 205 (1955).

[30] J. Collins, Foundations of Perturbative QCD, Vol. 32 (Cambridge University Press, Cambridge, England, 2013).

[31] J. C. Collins, Renormalization: An Introduction to Renormalization, The Renormalization Group, and the Operator Product Expansion, Cambridge Monographs on Mathematical Physics, Vol. 26 (Cambridge University Press, Cambridge, England, 1986).

[32] R. E. Cutkosky, Singularities and discontinuities of Feynman amplitudes, J. Math. Phys. (N.Y.) 1, 429 (1960).

[33] S. J. Brodsky, P. Hoyer, C. Peterson, and N. Sakai, The intrinsic charm of the proton, Phys. Lett. 93B, 451 (1980). 